\title{
PHARMACEUTICAL PROCESS VALIDATION: A REVIEW
}

\author{
Kaur Harpreet*, Singh Gurpreet, Seth Nimrata \\ Rayat Institute of Pharmacy, Railmajra,SBS Nagar,Punjab, India \\ *Corresponding Author’s E-Mail-kaurkhalsa88@gmail.com, Telephone no: 09041860980
}

\begin{abstract}
Validation is the art of designing and practicing the designed steps alongside with the documentation. Process validation emphasize on process designelements and maintaining process control during commercialization andcommunicate that process validation is an ongoing program and alignprocess validation activities with product lifecycle. Process validation alsoemphasizes the role of objective measures and statistical tools \& analysesand emphasizes knowledge, detection, and control of variability and givesassurance on consistent of quality/productivity throughout life cycle of product. According to GMP, validation studies are essential part of GMP these are required to be done as per predefined protocols. The process validation is establishing documented evidence which provides high degree on assurance that a specific process consistenly produced a product meeting its predetermined specifications and quality characteristic. The validation study provide the accuracy, sensitivity, specificity and reproducibility of the test methods employed by the firms, shall be established and documented. Thus the validation is an essential part of the quality assurance. Lending importance to validation is increasingly profound in recent years.
\end{abstract}

Keywords: Process Validation, GMP, specifications, consistent, documented.

\section{INTRODUCTION}

Pharmaceutical Process Validation is the most important and recognized parameters of cGMPs. The requirements of process validation appear of the quality system (QS) regulation. The goal of a quality system is to consistently produce products that are fit for their intended use. Process validation is a key element in assuring that these principles and goals are met. The process validation is standardization of the validation documents that must be submitted with the submission file for marketing authorization. The process validation is intended to assist manufacturers in understanding quality management system (QMS) requirements concerning process validation and has general applicability to manufacturing process. According to FDA, assurance of product quality is derived from careful and systemic attention to a number of importance factors, including: selection of quality process through in-process and end-product testing. Validation is a concept that has evolved inunite states in 1978. The concept of validation has expanded through the years to embrace a wide range of activities from analytical methods used for the quality control of drug substances and drug products to computerized systems for clinical trials, labelling or process control. Validation isfounded on, but not prescribed by regulatory requirements and is best viewed as an important and integral part of cGMP. The word validation simply means assessment of validity or action of proving effectiveness. Validation is a team effort where it involves people from various disciplines of the plant.

\section{WHY VALIDATION?}

- The pharmaceutical industry uses expensive material, sophisticated facilities and equipments and highly qualified personals.

-Detailed study and controlled of the manufacturing process batch validation is necessary if failure cost is to be reduced and productivity is improved.
- If would not be feasible to use equipment not knowing if it will produce the product we want, not to employ the people with no assurance that they can do or fail to implement process checks or examination to assure that product meet specifications.

- The efficient use of these resources is necessary for the continued success of the industry. The cost of product failure, rejects, reworks, recalls, complaints are the sufficient part of total production cost.

-Assurance of quality, cost reduction.

\section{MAJOR PHASES IN VALIDATION: ${ }^{3,4}$}

The activities relating to validation studies may be classified into three:

\section{Phase 1:}

This is the Pre-validation Qualification Phase which covers all activities relating to product research and development, formulation pilot batch studies, scale-up studies, transfer of technology to commercial scale batches, establishing stability conditions and storage, and handling of in-process and finished dosage forms, equipment qualification, installation qualification, master product document, operational qualification and process capacity.

\section{Phase 2:}

This is the Process Validation Phase. It is designed to verify that all established limits of the critical process parameter are valid and that satisfactory products can be produced even under the worst conditions.

\section{Phase 3:}

This is known as the Validation Maintenance Phase, it requires frequent review of all process related documents, including validation of audit reports, to assure that there have been no changes, deviations, failures and modifications to the production process and that all 
standard operating procedures (SOPs), including change control procedures, have been followed. At this stage, the validation team comprising of individuals representing all major departments also assures that there have been no changes/deviations that should have resulted in requalification and revalidation ${ }^{3}$. A careful design andvalidation of systems and processcontrols can establish a high degree ofconfidence that all lots or batches producedwill meet their intended specifications. Itis assumed that throughoutmanufacturing and control, operationsare conducted in accordance with theprinciple of good manufacturing practice(GMP) both in general and in specificreference to sterile product manufacture.

\section{VALIDATION STEPS:}

The validation steps recommended in GMP guidelines can be summarized as follows ${ }^{3}$ :

- As a pre-requisite, all studies should be conducted in accordance with a detailed, pre-established protocol or series of protocols, which in turn is subject to formal change control procedures.

- Both the personnel conducting the studies and those running the process being studied,should be appropriately trained and qualified and be suitable and competent to perform the task assigned to them.

- All data generated during the course of studies should be formally reviewed and certified as evaluated against predetermined criteria.

-Suitable testing facilities,equipment,instruments and methodology should be available.

- Suitable clean room facilities should be available in both the 'local' and background

environment. There should be assurance that the clean room environment as specified is secured through initial commissioning (qualification) and subsequently through the implementation of a programme of re-testinginprocess equipment should be properly installed, qualified and maintained.

-When appropriate attention has been paid to the above, the process, if aseptic, may be validated by means of "process simulation" studies.

-The process should be revalidated at intervals; and Comprehensive documentation should be available to define support and record the overall validation process.

\section{Planning for Validation}

All validation activities should be planned. The key elements of a validation programme should be clearly defined and documented in a validation master plan (VMP) or equivalent documents.

-The VMP should be a summary document, which is brief, concise and clear.

following:

-The VMP should contain data on at least the

1.Validation policy.

2.Organisational structure of validation activities.

3.Summary of facilities, systems, equipment and processes to be validated.

C 2011, JDDT. All Rights Reserved
4.Documentation format: The format to be used for protocols and reports.

5.Planning and scheduling.

6.Change control.

7.Reference to existing document.

8.Incase of large projects, it may be necessary to create separate validation master plans.

\section{Documentation}

A written protocol should be established that specifies how qualification and validation willbe conducted. The protocol should be reviewed and approved. The protocol should specify critical steps and acceptance criteria. A report that contain references, the qualification and/or validation protocol should be prepared, summarizing the results obtained, commenting on any deviations observed, and drawing the necessary conclusions, including recommending changes necessary to correct deficiencies. Any changes to the plan as defined in the protocol should be documented with appropriate justification. After completion of a satisfactory qualification, a format release for the next step in qualification and validation should be made as a written authorization.

\section{Validation set up}

To establish the desired attributes. These attributes include physical as well as chemical characteristics. In the case of parenterals, these desirable attributes should include stability, absence of pyrogens, and freedom from visible particles. Acceptance specifications for the product should be established inorder to attain uniformity and consistently the desired product attributes, and the specifications should be derived from testing and challenge of the system on sound statistical basis during the initial development and production phases and continuing through subsequent routine production. The process and equipment should be selected to achieve the product specification. For example; design engineers; production and quality assurance people may all be involved.The process should be defined with a great deal of specificity and each step of the process should be challenged to determine its adequacy. These aspects are important inorder to assure products of uniform quality, purity and performance.

\section{PROCESS VALIDATION}

"Process Validation is establishing documented evidence which provides a high degree ofassurance that a specific process will consistently produce a product meeting its pre-determined specifications and quality characteristics."

It is beneficial to the manufacturer in many ways: ${ }^{14}$

-It deepens the understanding of processes, decreases the risk, preventing problems and thus assures the smooth running of the process.

-It decreases the risk of defect costs.

-It decreases the risk of regulatory non- compliance.

-A fully validated process may require less in-process controls and end- product testing. 
Validation should thus be considered in the following situations:

-Totally new process.

-New equipment.

- Process and equipment which have been altered to suit changing priorities.

- Process where the end-product test is poor and an unreliable indicator of product quality.

\section{BASIC CONCEPT OF PROCESS VALIDATION}

-Calibration, verification and maintenance of process equipment.

•requalification or revalidation.

-Establishing specifications and performance characteristics.

-election of methods, process and equipment to ensure the product meets specifications.

-Qualification or validation of process and equipment.

- Testing the final product, using validated analytical methods, in order to meet specifications.

-Challenging, auditing, monitoring or sampling the recognised critical key steps of the process.

\section{Objectives of process validation}

- The manufacturing process, in addition to the individual equipment, must be validated.

- The goal is to create a robust manufacturing process that consistently produces a drug product with minimal variation that adheres to quality criteria of purity, identity, and potency.

- A validation plan for the manufacturing process should be drafted and executed by engineers in order to satisfy guidelines. The validation plan usually involves just a PQ section.

- Just as equipment validation, major changes after the initial validation will result in the need for subsequent revalidation.

- In the end, process validation will ensure a robust product that is highly reproducible over time.

Advantages of process validation

- Expanded real time monitoring and adjustment of process.

- Enhanced ability to statistically evaluate process performance and product variables. e.g., individuals; mean; range; control limits.

- Enhanced data and evaluation capabilities and increased confidence about process reproducibility and product quality.

- Improved ability to set target parameters and control limits for routine production, correlating with validation results.

- Enhanced reporting capability.

Reason for Process Validation

The possible reason of performing process validation may include:
- New product or existing products as per SUPAC changes .

- Change in site of manufacturing.

- Change in batch size.

- Change in equipment.

- Change in process existing products.

- Change in composition or components.

- Change in the critical control parameters.

- Change in vendor of API or critical excipient.

- Change in specification on input material.

- Abnormal trends in quality parameters of product through review during Annual Product Review (APR).

Trend of Out of Specification (OOS) or Out of Trend (OOT) in consecutive batches.

\section{$\underset{2,5,6,7]}{\text { BASIC PRNCIPLE FOR PROCESS VALIDATION: }}{ }^{[1,}$}

The basic principle for validation may be stated as follows:Installation Qualification (IQ):establishing by objective evidence that all key aspects of the process equipment and ancillary system installation adhere to the manufacturer's approved specification and that the recommendation of the supplier of the equipment are suitably considered.

\section{IQ considerations are:}

-Equipment design features (i.e. material of construction cleanability, etc.)

-Installation conditions (wiring, utility, functionality, etc.)

-Calibration, preventative maintenance,cleaning schedules.

-Safety features.

-Supplier documentation, prints, drawings and manuals.

-Software documented.

-Spare parts list.

-Environmental conditions (such as clean room requirements, temperature, and humidity).

Operational Qualification (OQ):Establishing by objective evidence process control limits and action levels which result in product that all predetermined requirements.

\section{OQ considerations include:}

-Process control limits (time, temperature, pressure, line speed, setup conditions, etc.)

-Software parameters.

- Raw material specifications.

-Process operating procedures.

-Material handling requirements.

- Process change control.

-Training.

- Short term stability and capability of the process, (latitude studies or control charts).

-Potential failure modes, action levels and worst-case conditions. 
-The use of statistically valid techniques such as screening experiments to optimize theprocess can be used during this phase.

Performance Qualification (PQ): establishing by objective evidence that the process, under anticipated conditions, consistently produces a product which meets all predetermined requirements.

\section{PQ considerations include:}

- Actual product and process parameters and procedures established in OQ.

- Acceptability of the product.

- Assurance of process capability as established in OQ.

- Process repeatability, long term process stability.

Re-Qualification:Modification to, or relocation of equipment should follow satisfactory review and authorization of the documented change proposal through the change control procedure. This formal review should include consideration of re-qualification of the equipment. Minor changes or changes having no direct impact on final or in-process product quality should be handled through the documentation system ofthe preventive maintenance program.

\section{TYPES OF PROCESS VALIDATION: ${ }^{1,8}$}

Prospective Validation:Conducted prior to the distribution of either a new product or a product made under a modified production process, where the modifications are significant and may affect the products characteristics. It is a preplanned scientific approach and includes the initial stages of formulation development,process development, setting of process specifications, developing in-process tests sampling plans, designing of batch records, defining raw material specifications, completion of pilot runs, transfer of technology from scale-up batches to commercial size batches, listing major process is executed and environmental controls. ${ }^{18}$ In Prospective Validation, the validation protocol is executed before the process is put into commercial use. It is generally considered acceptable that three consecutive batches/runs within the finally agreed parameters, giving product of the desired quality would constitute a proper validation of the process. It is a confirmation on the commercial three batches before marketing. ${ }^{[8]}$

Concurrent Validation:A process where current production batches are used to monitor

processing parameters. It gives of the present batch being studied, and offers limited assurance regarding consistency of quality from batch to batch. ${ }^{18}$ Concurrent Validation may be the practical approach under certain circumstances. Examples of these may be when: ${ }^{8}$

- A previous validated process is being transferred to a third party contract manufacturer or to another site.

- The product is a different strength of a previously validated product with the same ratio of active/inactive ingredients.

- The number of lots evaluated under the Retrospective Validation were not sufficient to obtain a high degree of assurance demonstrating that the process is fully under control.

- The numbers of batches produced are limited.

- Process with low production volume per batch and market demand.

- Process of manufacturing urgently needed drug due to shortage or absence of supply.

- In all above cases concurrent validation is valid, provided following conditions are appropriately.

-Pre-approved protocol for concurrent validation with rational.

- A deviation shall be raised with justification and shall be approved by plant head /head process owner/Head-QMS.

- Product behaviour and history shall be reviewed based on developmental/scale up /test batches.

- A detailed procedure shall be planned for handling of the marketed product if any adverse reactions observed in concurrent validation process.

- Concurrent validation batches shall be compiled in report and shall be approved all key disciplines.

Retrospective Validation:Conducted for a product already being marketed, and is based on extensive data accumulated over several lots and over time. Retrospective Validation may be used for older products which were not validated by the fabricator at the time that they were first marketed, and which are now to be validated to confirm to the requirements of division 2, Part $\mathrm{C}$ of the Regulation to be Food and Drugs Act. Retrospective Validation is only acceptable for well established detailed processes and will be Inappropriate where there have recent changes in the formulation of the products, operating procedures, equipment and facility ${ }^{18}$ Some of the essential elements for Retrospective Validation are: ${ }^{8}$

-Batches manufactured for a defined period (minimum of 10 last consecutive batches).

- Number of lots released per year.

-Batch size/strength/manufacturer/year/period.

- Master manufacturing/packaging documents.

- List of process deviations, corrective actions and changes to manufacturing documents.

-Data for stability testing for several batches.

-Trend analysis including those for quality related complaints.

Process Re-Validation:Required when there is a change in any of the critical process parameters, formulation, primary packaging components, raw material fabricator, major

equipment or premises. Failure to meet product and process specifications in batches would also require process re-validation. ${ }^{18}$

- Re-Validation becomes necessary in certain situations. The following are examples some of the planned or unplanned changes that may require re-validation: 
- Changes in raw materials (physical properties such as density, viscosity, particle size distribution, and moisture, etc., that may affect the process or product).

-Changes in the source of active raw material manufacturer.

-Changes in packaging material (primary container/closure system).

-Changes in the process (e.g., mixing time, drying temperatures and batch size).

- Changes in the equipment (e.g. addition of automatic detection system).

-Changes of equipment which involve the replacement of equipment on a "like for like"

basis would not normally require a revalidation except that this new equipment.

-Must be qualified.

-Changes in the plant/facility.

-Variations revealed by trend analysis (e.g. process drifts).

\section{STRATEGY FOR VALIDATION}

\section{VALIDATION PROTOCOL: ${ }^{5,9}$}

Detailed protocol for performing validations are essential to ensure that the process is adequately validated. Process validation protocols should include the following elements:

-Objectives, scope of coverage of the validation study.

-Validation team membership, their qualifications and responsibilities.

-Type of validation: prospective, concurrent, retrospective, re-validation.

- Number and selection of batches to be on the validation study.

-A list of all equipment to be used; their normal and worst case operating parameters.

- Outcome of IQ, OQ for critical equipment.

- Requirements for calibration of all measuring devices.

- Critical process parameters and their respective tolerances.

- Process variables and attributes with probable risk and prevention shall be captured.

-Description of the processing steps: copy of the master documents for the product.

-Sampling points, stages of sampling, methods of sampling, sampling plans.

-Statistical tools to be used in the analysis of data.

-Training requirements for the processing operators.

-Validated test methods to be used in inprocess testing and for the finished product.

-Specifications for raw and packaging materials and test methods.

- Forms and charts to be used for documenting results.
- Format for presentation of results, documenting conclusions and for approval of study results.

\section{VALIDATION OF ANALYTICAL METHODS. ${ }^{10}$}

Method validation confirms that the analytical procedure employed for a specific test is suitable for its intended use. The validation of an analytical method is the process by which it is established by laboratory studies that the performance characteristics of the method meet the requirement for the intended application. This implies that validity of a method can be demonstrated only though laboratory studies. ${ }^{15}$

Methods should be validated or revalidated: ${ }^{16,17}$

- before their introduction and routine use;

- whenever the conditions change for which the method has been validated, e.g., instrument with different characteristics; and

- wherever the method is changed and the change is outside the original scope of the method.

\section{ENVIRONMENTAL CONSIDERATIONS: \\ CLEANING AND CLEAN ROOM STANDARDS}

Cleaning validation is documented proof that one can consistently and effectively clean a system or equipment items. The procedure is necessary for the following reasons: ${ }^{10,11}$

-It is a customer requirement - it ensures the safety and purity of the product;

- It is a regulatory requirement in active pharmaceutical product manufacture;

-It also assures from an internal control and compliance point of view the quality of the process.

\section{INSPECTION OF EQUIPMENTS:}

The FDA guide to inspections ${ }^{12}$ intended to cover equipment cleaning (chemical residues only) expects firms to have written procedure (SOPs) detailing the cleaning processes and also written general procedure on how cleaning processes will be validated.

- A standard operating procedure (SOP) for cleaning with a checklist;

- A procedure for determining cleanliness (rinse or swab);

- An assay for testing residual drug levels;

-Pre-set criteria for testing chemical and microbial limit to which to equipment must be cleaned;

-Protocol for cleaning validation.

The cleaning protocol must be thorough and must be checked. Training is essential. A validation program requires

- criteria for acceptance after cleaning,

- appropriate methods of sampling

- a maximum limit set for residues, and

- test methods that must themselves be tested. 


\section{EXPERT EVALUATION}

This is an evaluation of the entire study against the protocol requirements as outlined above. It should be prepared and the conclusion drawn at each stage stated. The final conclusions should reflect whether the protocol requirements were met. The evaluation should include an assessment of the planned calibration and maintenance programmes for the equipment and instrumentation to maintain the validated conditions. In addition, all process monitoring and control procedures required to routinely ensure that the validated conditions are maintained should be reported. The evaluation should be signed by authorized officers of the organization who were members of the team establishing the protocol and who are appropriate expertise in the area assigned to them. Overall approval of the study should be authorized by the head of the validation team and the head of the quality control department. ${ }^{13}$

\section{THE VALIDATION REPORT}

A written report should be available after completion of the validation. If found acceptable, it should be approved and authorized (signed and dated). The report should include at least the following: ${ }^{19}$

- Title and objective of study;

- Reference to protocol;

\section{REFERENCES:}

1 Guidance for Industry: Process Validation:General Principles and Practices. U.S. Department of Health and Human Services, Food and Drug Administration, Centre for Drug Evaluation and Research (CDER), Centre for Biologics Evaluation and Research (CBER), Centre for Veterinary Medicine (CVM), January 2011.

2. Quality Management System - Process Validation Guidance GHTF/SG3/N99-10:2004 (Edition 2).

3. Elsie Jatto, Augustine and O. Okhamafe; An Overview of Pharmaceutical Validation andProcess Controls in Drug Development, Tropical Journal of Pharmaceutical Research, December 2002; 1 (2): 115-122.

4. Guide to Inspections of Oral Solid Dosage Forms Pre/Post Approval Issued for Development and Validation. Washington DC: US Food and Drug Administration, 1994.

5. Health Canada / Health Products and Food Branch Inspectorate Validation Guidelines forPharmaceutical Dosage Forms (GUI 0029) / December, 2009.

6. Validation Master Plan Installation and Operational Qualification -PharmaceuticalInspection Convention; Pharmaceutical Inspection Co-Operation Scheme; PI 006 - 2; July,2004.

7. Kathiresan K*, Moorthi C, Prathyusha Y, Gade B. R, Reddy B. K, Manavalan R, ; An overview of pharmaceutical validation; Research Journal of Pharmaceutical, Biological and Chemical Sciences; ISSN: 0975-8585; October - December 2010; RJPBCS 1(4); Page No. 1026.

8. Guidelines for Process Validation of Pharmaceutical Dosage Form - Saudi Food \& Drug Authority; Version 2; February, 1992.
-Details of material;

-Equipment;

-Programmes and cycles used;

-Details of procedures and test methods;

- Results (compared with acceptance criteria); and

- Recommendations on the limit and criteria to be applied on future basis.

\section{CONCLUSION}

From the study it can be stated that pharmaceutical Process Validation is the most important and recognized parameters of cGMP. The cGMP regulation require that manufacturing processes be designed and controlled to assure that in-process materials and finished productmeet predetermined quality requirements and do so consistently and reliably. The productshould be designed robustly enough to withstand variations in the manufacturing process and the manufacturing process should be capable and stable to assure continued safe products thatperform adequately. Process validation involves a series of activities taking place over thelifecycle of the product and process.

9. Guide to Inspections of Oral Solid Dosage Forms pre/post Approval Issue for Development and Validation; issue (1/94); January, 2010.

10. Green JM. A Practical Guide to Analytical Method Validation, Anal. Chem. News andFeatures 1996; 60:305A-9A.

11. Akers, J. Simplifying and improving process validation. J. Parent. Sci. Technol. 1993, 47, 281-284.

12.Avallone, H.L.; D'Eramo, P. Scale-up and validation of ANDA/NDA products. Pharm. Eng.1992, 12 (6), 36-39.

13.Chowhan, Z.T. Development of a new drugsubstance into a compact tablet. Pharm. Technol.1992, 16 (9), 58-67.

14. Nash RA. Process Validation of a 17-Year retrospective study of solid dosage forms.DrugDevInd Pharm 1966; 22 (1): 25-34.

15. Therapeutics Products Programme. Process Validation: Aseptic Processes for Pharmaceuticals.http://www.hc-sc.gc.ca/hpbdgps/ therapeutic; downloaded March 30, 2001.

16. Validation of Compendia Methods. United States Pharmacopoeia and National Formulary XVIII, Rockville, MD: The United States Pharmacopoeia Convention, Inc., 1995, pp. 1612-710.

17. Validation of Analysis Procedures.International Conference on Harmonization (ICH) of Technical

18. Health Canada / Health Products and Food Branch Inspectorate Validation Guidelines forPharmaceutical Dosage Forms (GUI 0029) / December, 2009.

19. Good Manufacturing Practices for Pharmaceutical Products. WHO Expert Committee on Specifications for Pharmaceutical Preparations.32nd Report, WHO Technical Report Series no. 823. Geneva: WHO, 1992: pp 14-96. 